\title{
Why the aortic dissection detection risk score is problematic in emergency departments
}

\author{
Anne-Maree Kelly ${ }^{1,2,3}{ }^{*}$ (]) \\ ${ }^{1}$ Joseph Epstein Centre for Emergency Medicine Research at Western Health, Melbourne 3021, Australia \\ ${ }^{2}$ Department of Medicine, Western Health, Melbourne 3021, Australia \\ ${ }^{3}$ Melbourne Medical School, the University of Melbourne, Melbourne 3010, Australia \\ *Correspondence: Anne-Maree Kelly, JECEMR, Sunshine Hospital, Furlong Road, St Albans 3021 VIC Australia. \\ Anne-Maree.Kelly@wh.org.au \\ Academic Editor: Kathleen G. Morgan, Boston University, USA \\ Received: May 5, 2021 Accepted: July 30, 2021 Published: August 31, 2021
}

Cite this article: Kelly AM. Why the aortic dissection detection risk score is problematic in emergency departments. Explor Med. 2021;2:348-51. https://doi.org/10.37349/emed.2021.00053

\begin{abstract}
Acute aortic syndromes, including aortic dissection (AD), are rare. The AD detection risk score (ADDRS) and associated investigation pathway were developed to reduce missed diagnosis of AD. The methodology for its development was sub-optimal and it has not been robustly validated in the emergency department chest pain population. Recent research suggests that it will drive over-investigation and that the risks of missed diagnosis may not be in balance with the risks of the testing strategy. There are serious doubts about whether the score and investigation pathway are fit for purpose.
\end{abstract}

\section{Keywords}

Risk score, aortic dissection, clinical decision rule

Acute aortic syndromes, including aortic dissection (AD), are rare. Presentation is variable and misdiagnosis is common [1]. In those that survive to reach hospital, chest pain is a common, but not universal, symptom [2]. Even among emergency department (ED) chest pain patients, AD is rare. Using data from the United States, AD was found to be present in approximately 1:12,000 ED visits and approximately 1:980 patients presenting with atraumatic chest pain [2]. It is not known whether this is representative of experience in other countries.

Using consensus methodology and registry data, the American Heart Association, in collaboration with other stakeholders (but not emergency physicians), developed the AD detection risk score (ADDRS) and an associated investigation pathway [3]. Its aim was to reduce the rate of misdiagnosis. The score, out of three, allocates one point each for the presence of high-risk conditions, high-risk pain features and high-risk examination features. High risk conditions are defined as Marfan's syndrome, family history of aortic disease, known aortic valve disease and known thoracic aortic aneurysm. High risk pain is defined as chest, back or abdominal pain that is either of abrupt onset or severe intensity or ripping/tearing in character. High risk examination features are defined as evidence of a perfusion deficit, focal neurological signs or new artic

(C) The Author(s) 2021. This is an Open Access article licensed under a Creative Commons Attribution 4.0 International License (https://creativecommons.org/licenses/by/4.0/), which permits unrestricted use, sharing, adaptation, distribution and reproduction in any medium or format, for any purpose, even commercially, as long as you give appropriate credit to the original author(s) and the source, provide a link to the Creative Commons license, and indicate if changes were made. 
insufficiency in the presence of pain or hypotension/shock [3]. The score was designed to apply to all chest pain patients.

The associated diagnostic pathway recommends D-dimer testing for scores $\leq 1$ (including 0 ) and computed tomography angiography (CTA) for scores $>1$, with positive D-dimer results $(>500 \mathrm{ng} / \mathrm{mL})$ also proceeding to CTA.

In effect, the ADDRS and associated pathway is promoted as a clinical decision rule for use in ED. Unfortunately, it was not developed using methodology that would be widely accepted for development of such rules [4]. The key weakness is that the variables chosen for inclusion in the score have not been adequately tested for their discriminative performance in ED chest pain patients. Further, the relative weight assigned to the variables does not appear to be based on statistical analyses. Additionally, there has not been robust validation on the population of interest-the undifferentiated ED chest pain population. The lens of those who developed the ADDRS was that of those who image or treat AD. Unfortunately, those groups did not have broad experience of chest pain in ED.

Previous research has focussed on establishing the sensitivity of the score and pathway, which is high-99.9\% in a recent systematic review [5]. Little attention has been given to specificity of the score. The majority of studies have been limited to patients in whom AD was suspected or identified as a differential diagnosis. They therefore exclude patients in whom AD was not considered or was considered so unlikely that inclusion as a differential diagnosis was not thought clinically justified. Medical litigation has identified these reasons as contributors to missed diagnosis of $\mathrm{AD}[6]$.

There has been very limited research on the impact of broad application of the score and pathway. For example, it could result in increased rates of testing, especially CTA, with its inherent risks. If the proportion of patients with $\mathrm{AD}$ is very low, this could result in more harm than good.

A recent study explored the distribution of ADDRS in undifferentiated chest pain patients and estimated the impact on testing that would flow if the recommended investigation strategy was applied to patients with a final ED diagnosis of non-specific chest pain, i.e. after other causes had been excluded [7]. It found that $23.7 \%$ of chest pain patients had a score of $0,72.7 \%$ had a score of 1 and $3.6 \%$ had a score of 2 . No patients had a score of 3 . No patients reported high risk conditions associated with AD. High-risk pain features were present in $75.5 \%$ of patients. High risk examination features were present in $4.3 \%$ of patients. Of patients with an ADDRS score of 1,99\% were due to patient reported pain features alone. No patient was diagnosed with AD. The estimated impact of the implementation of the ADDRS score investigation pathway in patients with a final ED diagnosis of non-specific chest pain was a 280\% increase in D-dimer testing and a 2,200\% increase in CTA testing. It was estimated that $23 \%$ of ED patients with non-specific chest pain would require a CTA, compared to the estimated prevalence of $\mathrm{AD}$ in the ED chest pain population of $0.1 \%$ [2]. This prevalence is probably over-estimated, because its derivation set would have included patients with high-risk conditions and high-risk examination features-characteristics not usually present in patients with a final ED diagnosis of non-specific chest pain.

If it is assumed that the risk of missed $A D$ after an ED assessment is somewhere between 1:1,000 and 1:5,000, this needs to be balanced against the risks associated with CTA. The risk of cancer risk from radiation associated with CTA (fatal or non-fatal) is age dependent. It has been estimated for males to be at 1:636 at age 40 and 1:860 at age 60 . For women, the estimated risk is 1:590 for 40-year-olds and 1:890 for 60-year-olds respectively [8]. The risk is higher for younger people. The risk of life-threatening, severe allergic reaction from contrast administration is estimated at 1:1,000, with a 1:75,000 risk of death [9]. There is also a small risk of contrast nephropathy and risks associated with the discovery of incident findings. This risk balancing analysis suggests that, for patients in whom pain features are the only 'risk factor', application of the ADDRS investigation pathway poses more risks than benefits. Even if the score was $100 \%$ sensitive in chest pain patients, there would still be missed AD, predominantly from the approximate $20 \%$ of cases who do not report chest pain [2].

There is no evidence about what risk of missed AD ED chest pain patients would reasonably accept. The estimated risk of missed myocardial infarction (MI)—a condition approximately 100 times more prevalent 
as a cause of chest pain in ED than AD-with contemporary acute coronary syndrome rule out processes is $<1 \%$ [10]. A survey of emergency physicians found that most (57\%) would accept a risk of major adverse clinical events (MACE) due to missed MI of 1:500 or less [11]. Their opinion is likely to be similar for missed AD. It would be helpful to know the opinions of patients with non-specific chest pain on this issue.

An obvious question is whether a better clinical decision rule could be developed. To achieve $100 \%$ sensitivity with confidence interval $97-100 \%$ would require 120 positive cases. With the known prevalence of AD in ED chest pain patients of approximate 1:1,000, any derivation study would require about 120,000 patients with a similar number for validation studies. Unfortunately, this is not feasible. In addition, patients with non-chest pain presentations of AD might still be missed.

Another approach is identification of more accurate biomarkers. This is a focus of current research $[12,13]$. None of these has been validated in ED clinical practice to date. To be effective in reducing missed $A D$, they would require high sensitivity and negative predictive value, accessibility in ED and a short turnaround time for results. Additionally, their impact on the ordering of CTA would need to be such that the risks of adverse events associated with CTA and missed diagnosis of AD were at least in balance. Having more accurate biomarkers does not overcome the problem that the biggest contributors to missed diagnosis of $\mathrm{AD}$ is failure to suspect $\mathrm{AD}$ and atypical symptoms.

In conclusion, the ADDRS score and associated investigation strategy are not fit for purpose for use in ED patients with non-specific chest pain due to its methodological weaknesses. It also does not appropriately balance risks, especially for younger patients. Consequently, its application to ED patients with non-traumatic chest pain cannot be justified.

\section{Abbreviations}

AD: aortic dissection

ADDRS: aortic dissection detection risk score

CTA: computed tomography angiography

ED: emergency department

\section{Declarations}

Author contributions

The author contributed solely to the work.

\section{Conflicts of interest}

The author declares that she has no conflicts of interest.

Ethical approval

Not applicable.

\section{Consent to participate}

Not applicable.

\section{Consent to publication}

Not applicable.

Availability of data and materials

Not applicable.

\section{Funding}

Not applicable. 


\section{Copyright}

(C) The Author(s) 2021.

\section{References}

1. Chua M, Ibrahim I, Neo X, Sorokin V, Shen L, Ooi SB. Acute aortic dissection in the ED: risk factors and predictors for missed diagnosis. Am J Emerg Med. 2012;30:1622-6.

2. Alter SM, Eskin B, Allegra JR. Diagnosis of aortic dissection in emergency department patients is rare. West J Emerg Med. 2015;16:629-31.

3. Hiratzka LF, Bakris GL, Beckman JA, Bersin RM, Carr VF, Casey DE Jr, et al. 2010 ACCF/AHA/AATS/ACR/ ASA/SCA/SCAI/SIR/STS/SVM guidelines for the diagnosis and management of patients with thoracic aortic disease: a report of the American College of Cardiology Foundation/American Heart Association Task Force on Practice Guidelines, American Association for Thoracic Surgery, American College of Radiology, American Stroke Association, Society of Cardiovascular Anesthesiologists, Society for Cardiovascular Angiography and Interventions, Society of Interventional Radiology, Society of Thoracic Surgeons, and Society for Vascular Medicine. Circulation. 2010;121:e266-369.

4. Stiell IG, Wells GA. Methodologic standards for the development of clinical decision rules in emergency medicine. Ann Emerg Med. 1999;33:437-47.

5. Bima P, Pivetta E, Nazerian P, Toyofuku M, Gorla R, Bossone E, et al. Systematic review of aortic dissection detection risk score plus D-dimer for diagnostic rule-out of suspected acute aortic syndromes. Acad Emerg Med. 2020;27:1013-27.

6. jade.io [Internet]. Sydney: JADE; c2021 [cited 2021 May 5]. Available from: https://jade.io/ article/696992?at.hl=boxell

7. Rawicki AJ, Klim S, Kelly AM. What is the distribution of aortic dissection detection risk score in an undifferentiated emergency department chest pain population? Emerg Med Australas. 2020;32:872-4.

8. Smith-Bindman R, Lipson J, Marcus R, Kim KP, Mahesh M, Gould R et al. Radiation dose associated with common computed tomography examinations and the associated lifetime attributable risk of cancer. Arch Intern Med. 2009;169:2078-86.

9. Saljoughian M. Intravenous radiocontrast media: a review of allergic reactions. US Pharm. 2012;37:HS-14-16.

10. Cullen L, Mueller C, Parsonage WA, Wildi K, Greenslade JH, Twerenbold R, et al. Validation of highsensitivity troponin I in a 2-hour diagnostic strategy to assess 30-day outcomes in emergency department patients with possible acute coronary syndrome. J Am Coll Cardiol. 2013;62:1242-9.

11. Than M, Herbert M, Flaws D, Cullen L, Hess E, Hollander JE, et al. What is an acceptable risk of major adverse cardiac event in chest pain patients soon after discharge from the Emergency Department? A clinical survey. Int J Cardiol. 2013;166:752-4.

12. Wang Y, Tan X, Gao H, Yuan H, Hu R, Jia L, et al. Magnitude of soluble ST2 as a novel biomarker for acute aortic dissection. Circulation. 2018;137:259-69.

13. Cheng M, Yang Y, Xin H, Li M, Zong T, He X, et al. Non-coding RNAs in aortic dissection: from biomarkers to therapeutic targets. J Cell Mol Med. 2020;24:11622-37. 